\title{
GIC: A computational method for predicting the essentiality of long noncoding IncRNAs
}

$$
\text { Pan Zeng", Ji Chen", Yuan Zhoü, Jichun Yang }{ }^{*} \text {, and Qinghua Cui* }
$$

Department of Biomedical Informatics, Department of Physiology and Pathophysiology,

Centre for Noncoding RNA Medicine, MOE Key Lab of Cardiovascular Sciences, School of

Basic Medical Sciences, Peking University, 38 Xueyuan Rd, Beijing, 100191, China

\# These authors contributed equally to this work.

* To whom the correspondence should be addressed:

Qinghua Cui, email: cuiqinghua@bjmu.edu.cn

or

Jichun Yang, email: yangj@ bjmu.edu.cn 


\section{ABSTRACT}

Measuring the essentiality of genes is critically important in biology and medicine. Some bioinformatic methods have been developed for this issue but none of them can be applied to long noncoding RNAs (IncRNAs), one big class of biological molecules. Here we developed a computational method, GIC (Gene Importance Calculator), which can predict the essentiality of both protein-coding genes and IncRNAs based on RNA sequence information. For identifying the essentiality of protein-coding genes, GIC is competitive with well-established computational scores. More important, GIC showed a high performance for predicting the essentiality of IncRNAs. In an independent mouse IncRNA dataset, GIC achieved an exciting performance (AUC=0.918). In contrast, the traditional computational methods are not applicable to IncRNAs. As a public web server, GIC is freely available at http://www.cuilab.cn/gic/. 


\section{INTRODUCTION}

Essential genes constitute a small fraction in a genome of an organism. However, these genes underpin numerous core biological processes and are indispensable for cell viability. Insufficient expression of essential genes will lead to increased vulnerability and loss-of-function mutations of essential genes often cause lethal phenotypes (Korona, 2011; Peters, et al., 2016). Hence the classification of genes as either essential or non-essential for organism survival has a profound influence on the study of molecular basis of various biological process (Wang, et al., 2015), disease genes, drug targets, and genome design (Liu, et al., 2015). In recent years, efficient gene knockout or knockdown by CRISPR/Cas9 and RNAi have been widely used to systematically evaluate the essentiality of genes and lncRNAs(Evers, et al., 2016; Morgens, et al., 2016) in whole organisms (Peters, et al., 2016) and human cells (Blomen, et al., 2015; Wang, et al., 2015; Wang, et al., 2014; Zhou, et al., 2014; Zhu, et al., 2016). These studies provided great helps in identifying functionally important genes and thus have great potential in discovering new genes for disease therapy and diagnosis (Tzelepis, et al., 2016). However, the problem is that these techniques are normally time and labor consuming and hard to be applied to mammals in a large-scale.

Therefore, computational methods have been developed as an effective complement of the experimental approaches. Sequence conservation score measured by comparative genomics and degree in a protein-protein interaction (PPI) network were proposed to evaluate gene essentiality based on the observation that these metrics show significant correlations with gene essentiality (Liang and Li, 2007). In addition, machine learning based method was also developed (Cheng, et al., 2013; Deng, et al., 2011; Seringhaus, et al., 2006). Moreover, more 
complex topology features of PPI network are also be used for the prediction of essential genes(del Rio, et al., 2009; Gatto, et al., 2015; Li, et al., 2016; Li, et al., 2015; Peng, et al., 2015). More recently, a nucleotide-based computational method was developed for the prediction of human essential genes(Guo, et al., 2017). These methods provided great helps for identifying essential protein-coding genes. Nevertheless, these methods require attributes discriminating essential genes, e.g. gene expression, conservation, sequence features, gene ontology (GO) annotation, protein domain, protein subcellular location, and interaction network topological properties. Therefore, the problem is that these methods often fail to predict the essentiality of lncRNAs, a big class of RNA molecules identified recently in human genome(Iyer, et al., 2015; Zhao, et al., 2016). The reason is that information needed by these methods is usually unavailable for lncRNAs because most lncRNAs show low sequence conservation, low expression level, and high specificity (Iyer, et al., 2015). Moreover, for most of the lncRNAs, information such as the ontology, subcellular location, and interaction network topological properties are still not available (Iyer, et al., 2015). In addition, although Pheg(Guo, et al., 2017) is designed based on RNA sequences but it did not work well on lncRNAs. The reason could be it is based on the CDS sequences of an mRNA.

Here, we developed GIC (Gene Importance Calculator), an algorithm that can efficiently quantify the essentiality of lncRNAs. In addition, besides lncRNAs, GIC also works on protein-coding genes. The results showed that GIC has good performance in predicting essential protein-coding genes. More importantly, GIC showed a high performance in predicting essential lncRNAs, whereas other methods can not be applied to lncRNAs. According to our knowledge, GIC is the first computational method to predict essential 
lncRNAs. GIC web server and the source code are freely available at http://www.cuilab.cn/gic.

\section{MATERIALS AND METHODS}

Datasets of RNA sequences. We downloaded human (GRCh37/hg19; Nov 9, 2014) and mouse (GRCm38/mm10; Jan 8, 2015) mRNA sequences deposited in the UCSC Table Browser (Karolchik, et al., 2004). Human and mouse lncRNA transcripts were downloaded from the NONCODE database (Zhao, et al., 2016) (version 4) and the sequences longer than $200 \mathrm{nt}$ were retained.

Datasets of essential genes. We retrieved human and mouse essential protein coding genes from DEG(Luo, et al., 2014) (version 10). In addition, we collected 7 mouse essential lncRNAs and 7 non-essential lncRNAs with experimental evidence as an independent testing set. These lncRNAs were annotated according to the Mouse Genome Informatics (MGI) database (Bello, et al., 2015; Bult, et al., 2016) (http://www.informatics.jax.org/phenotypes.shtml) and the results from Sauvageau et al.'s assays (Sauvageau, et al., 2013). Gene CRISPR/Cas9 scores in the KBM7 cell line were obtained from Wang et al.' study (Wang, et al., 2015).

RNA sequence features. The first feature is RNA sequence length. Then, with a sliding window of length 3 and step size of 1, we counted the number of times each of the 64 nucleotide triplets (e.g., ACT, GCC) occurred $c_{i}$ and converted it to frequency $f_{i}$ by the following formula.

$$
f_{i}=\frac{c_{i}}{\sum_{i=1}^{64} c_{i}}, i=1,2, \cdots, 64
$$


Besides, we used RNAfold (Hofacker, et al., 1994) (version 1.8.5) to predict RNA secondary structure with default parameters and calculated the minimum free energy (MFE) of the secondary structure. Given that longer RNAs favor lower energy state, we introduced here normalized MEF (nMFE) as follows,

$$
\mathrm{nMFE}=\frac{\mathrm{MFE}}{L}
$$

where $L$ is RNA sequence length. We then mapped the RNA sequence features to their corresponding genes. For genes with multiple transcripts, we used the mean value in subsequent analysis. The ID mapping files was retrieved from the Ensembl database (Yates, et al., 2016) (release 83) with the R/Bioconductor package biomaRt (Durinck, et al., 2009) and manually curated.

Logistic regression model and GIC score. To reduce the number of features, especially nucleotide triplet features, we ranked the nucleotide triplet features according to their individual AUC and retained only the top five nucleotide triplet features (CGA, GCG, TCG, ACG, TCA; the same for both human and mouse) without severe co-linearity problem (Pearson correlation $<0.8$ ) with other nucleotide triplet features. Moreover, considering that negative samples greatly outnumbered positive samples in the training set, a subset of negative samples was randomly selected to keep a 1:1 positive-to-negative ratio in the training dataset. Nevertheless, all negative samples were retained in the testing datasets in order to reflect the realistic performance of GIC score. After that, logistic regression models were constructed and cross validated for human and mouse genes and mouse lncRNAs separately. The logistic regression model is that 


$$
\begin{gathered}
\theta(p)=\beta_{0}+\beta_{1} L+\beta_{2} \mathrm{nMFE}+\sum \beta_{i} f_{i}, i=\mathrm{CGA}, \mathrm{GCG}, \mathrm{TCG}, \mathrm{ACG}, \mathrm{TCA} \\
\text { where } \theta(p)=\operatorname{logit}(p)=\ln \frac{p}{1-p}
\end{gathered}
$$

$\beta \mathrm{s}$ are the coefficients of corresponding model and $p$ is the conditional probability that a gene is essential $(Y=1)$. Accordingly, we defined the GIC score as the probability output $p$ of the corresponding logistic regression model. That is

$$
\text { GIC score }=p=\frac{1}{1+e^{-\theta(p)}}
$$

\section{Correlation analysis between GIC score and well-established measures of essential genes.}

To explore the relationship between GIC score and several known measures of essential genes, we downloaded corresponding datasets described in detail below and got the intersections of GIC scores and each of them. To assess gene persistence, we counted the homolog number for each gene using data from the Homologene database (NCBI Resource Coordinators., 2016) (build 68). To evaluate sequence conservation, we retrieved the $\mathrm{dN} / \mathrm{dS}$ ratio of each one-to-one mouse-human (and human-mouse) ortholog pair from the Ensembl database (release 83). The interaction network degrees were derived from the protein-protein interactions recorded in the BioGRID database (Stark, et al., 2006) (release 3.4.135). At last, genes were sorted by GIC score and median-binned into 200 bins for clearer illustration.

\section{Comparing the accuracy of human and mouse essential gene prediction. Gene}

essentiality was annotated as a Boolean value based on the corresponding essential gene set acquired from DEG. Using the R package pROC (Robin, et al., 2011), the ROC curves were plotted and the AUC values for GIC score and the abovementioned measures were calculated and compared. Note that only the samples for which all of the above-mentioned measures 
were available were used during the comparison.

Cell culture. Human T/G HA VSMC Cells were cultured in DMEM medium supplemented with $15 \%$ FBS, 2mM L-glutamine, $100 \mathrm{U} / \mathrm{mL}$ penicillin, and $10 \mathrm{mg} / \mathrm{mL}$ streptomycin. The media were renewed twice a week. All experimental procedures were conducted within a $\mathrm{CO}_{2}$ incubator at a temperature of $37^{\circ} \mathrm{C}$, in an atmosphere of $95 \%$ air and $5 \% \mathrm{CO}_{2}$.

siRNA knockdown of target mRNAs in T/G HA VSMCs. T/G HA VSMCs (vascular smooth muscle cells) with the confluence of $60 \%$ were synchronized with serum-free starvation for 24 hours, and then transfected with siRNA mixtures against various mRNAs (50nM) or scrambled siRNA (50nM) using VigoFect transfection kit (Vigorous Biotechnolog, Cat No T001) for 48 hours. The siRNA mixture was transfected into T/G HA VSMC cells according to the manufacturer's siRNA gene silencing protocol. Basically, the siRNAs against each target mRNA were the mixture of four sets of sequences according to different part of target mRNA. All the siRNA sequences were designed and synthesized by Beijing Biolino Inc. All the siRNA sequences against various target mRNAs were provided in Supplementary Table S1. The scrambled siRNA was also provided by Beijing Biolino Inc.

Real time PCR analysis of target mRNAs after siRNA transfection. T/G HA VSMCs were transfected with $50 \mathrm{nM}$ siRNAs or scrambled siRNAs as detailed above. Forty eight hours post transfection, total cellular RNA was extracted using the Trizol reagent according to the manufacturer's instructions. $0.5-1.0 \mu \mathrm{g}$ of total RNA was used for the reverse transcription reaction. Quantitative real time PCR was performed using the DNA Engine with Chromo 4 Detector (MJ Research,Waltham, MA). The relative expression of target genes in various 
groups were calculated using $2^{-\Delta \Delta \mathrm{Ct}}$ methodology. $\beta$-actin mRNA was used as housekeeping gene in the current study. All primer sequences used for real-time PCR assays were listed in

\section{Supplementary Table S2.}

Cell viability assay. Cell viability was measured by MTT assay as detailed previously. In brief, T/G HA VSMCs were seeded and transfected in 24-well plates. At 48 hours post siRNA transfection, MTT was added into the culture medium to the final concentration of $0.5 \mathrm{mg} / \mathrm{ml}$, and then the cells were incubated for 4 hour at $37^{\circ} \mathrm{C}$ in incubator. The culture medium was removed, and cells were lysed by gently rotating in 250uL DMSO for 30 minutes in darkness at room temperature. The absorbance at 490nm was measured using an automatic plate reader. In each experiment, 3-4 observations were set and determined for each siRNA mixture. The average absorbance reflected cell viability with the data normalized to the control group.

Flow cytometry analysis of cell apoptosis. At 48 hours post transfection, T/G VSMC cells were managed using Apoptosis Detection Kit with 7-AAD according to the manufacturer's protocol (Biolegend). In brief, the cells were washed with ice-cold PBS twice, and then resuspended in $100 \mu \mathrm{l}$ of Annexin $\mathrm{X}$ binding buffer (10mM HEPES, pH 7.4, 140mM NaCl, $1 \mathrm{mM} \mathrm{MgCl} 2,5 \mathrm{mM} \mathrm{KCl}$, and2.5 mM CaCl2), and then added $1 \mu \mathrm{l}$ FITC Annex $\mathrm{V}$ and $1 \mu \mathrm{l}$ 7-Add viability staining solution. The cells were incubated at room temperature in darkness for 15 minutes, and then were analyzed by FACScan analysis with Cellquest software (Becton Dickinson).

Code availability. GIC is implemented in Python and it relies on the external program RNAfold. We provide convenient online service on our GIC web server 
(http://www.cuilab.cn/gic). However, as for large RNAs or batch jobs, we recommend users download the source code on this server. Besides, the pre-calculated GIC scores of human and mouse genes, including both mRNAs and lncRNAs, are also available on the server.

\section{RESULTS AND DISCUSSION}

\section{The construction of GIC}

In brief, we managed to construct a logistic regression model (GIC) by integrating several features that can be derived from RNA sequences or predicted RNA secondary structures for measuring gene essentiality. First of all, the length of a RNA sequence was considered as a feature of gene essentiality based on the observation that RNAs encode conserved proteins are longer than those encode proteins with less conservation(Lipman, et al., 2002). And then we integrated the frequencies of some specific nucleotide triplets into the model. In addition, we found mRNA products of essential genes often form more stable structures, which are found to influence gene expression (Wan, et al., 2014). Thus, we utilized RNAfold (Hofacker, et al., 1994) to predict RNA secondary structure and its minimum free energy (MFE). Given that longer RNAs normally have lower MFE than shorter RNAs, we normalized MFE by sequence length in the model. Finally, given the serious imbalance between the numbers of essential genes and non-essential genes, we randomly selected a subset of negative samples (non-essential genes) to keep a balanced positive-to-negative ratio in the training dataset and trained the logistic regression model based on the balanced dataset. GIC score was defined as the probability output of the model.

\section{Comparison of GIC method with previous computational methods and experimental}




\section{methods on protein-coding genes}

We first tested GIC score on protein-coding genes. Because we aim to develop a method for predicting essential lncRNAs, we did not compare GIC with each existing methods. For simplicity, we only tested GIC score with three well established scores, protein network degree, dn/ds score, and homolog number score. We observed significant correlations between human GIC scores and other computational scores (Figure 1a-c; Spearman $\rho=0.67$, $\mathrm{P}=6.17 \times 10^{-27}$ with homolog number, Spearman $\rho=-0.92, \mathrm{P}=0$ with $\mathrm{dN} / \mathrm{dS}$, Spearman $\rho=$ $0.69, \mathrm{P}=4.51 \times 10^{-30}$ with protein interaction network degree, respectively). For mouse genes, we got similar results (Figure 2a-c).

Furthermore, we took the human and mouse essential genes stored in the DEG database as the benchmarks to evaluate the accuracy of GIC score. First, we ranked the human and mouse genes by GIC score and simply divided them into ten equal groups, respectively. Indeed, essential genes were enriched in groups of genes with higher GIC scores for both human (Figure 1d; $\mathrm{P}=1.31 \times 10^{-69}$, Pearson's Chi-squared test) and mouse (Figure 2d; $\mathrm{P}=$ $7.80 \times 10^{-68}$, Pearson's Chi-squared test).

In addition, in recent years, genetics-based methods such as CRISPR/Cas9 have been used for identifying essential genes in a specific condition, for example some cancer cell line. To test whether CRISPR/Cas9 scores in one condition can be useful in other conditions, we also compared GIC score with CRISPR/Cas9 scores. As a result, in terms of performance on the area under the receiver operator characteristic (ROC) curve (AUC), GIC score has an AUC of 0.675), whereas CRISPR/Cas9 scores in KBM7 cell line (Wang, et al., 2015) has an AUC of 
0.568 (Figure 3a), suggesting that CRISPR/Cas9 scores in one condition could be not useful for evaluating the essentiality of genes in other conditions. Moreover, GIC score showed competitive performance compared with the three well-established computational scores including homolog number $\left(\mathrm{AUC}=0.622, \mathrm{P}=2.76 \times 10^{-11}\right.$, bootstrap test $)$, the $\mathrm{dN} / \mathrm{dS}$ score of mouse-human 1-to-1 homolog $\left(\mathrm{AUC}=0.639, \mathrm{P}=2.57 \times 10^{-5}\right.$, bootstrap test $)$ and protein interaction network degree $\left(\mathrm{AUC}=0.647, \mathrm{P}=1.14 \times 10^{-3}\right.$, bootstrap test $)$.

\section{Performance of GIC method on predicting the essentiality of IncRNAs}

Next, we directly tested if GIC score is feasible to predict essential lncRNAs. To this end, we gleaned 14 mouse lncRNAs, of which seven were essential and the others were non-essential in mutagenesis assays, as an independent testing dataset (Methods). On this testing lncRNA dataset, GIC score showcased an AUC of 0.918 (Figure 3b and Supplementary Table S3). The outcome again verified the viability of GIC score. Currently, there is no specific tool for essential lncRNA prediction, mainly due to the special characteristics of lncRNAs. Our GIC score can measure lncRNA essentiality with RNA sequence only and will serve as a promising tool to prioritize functionally important lncRNAs. Given that Pheg also only needs sequences as the input data, here we compared GIC with Pheg. For each lncRNA, we first inputed its whole lncRNA sequence. Because Pheg works on mRNA CDS regions, we run Pheg using the putative maximum CDS sequence, which was predicted using ORF Finder (https://www.ncbi.nlm.nih.gov/orffinder/). As a result, Pheg did not achieve a comparable performance for both whole sequences $(\mathrm{AUC}=0.531$ ) and $\mathrm{CDS}$ sequences $(\mathrm{AUC}=0.653$,

\section{Figure 3b).}




\section{A case study of using GIC to select genes essential in cancer but non-essential in other}

cells

Genome-level gene knockout in cancer cell lines provides great helps in identifying essential genes in given cells (Wang, et al., 2015). These essential genes could be candidate targets for cancer therapy. For specificity of therapy, one ideal condition is that these essential genes are non-essential in other types of normal cells. This makes it important to identify these genes that are essential in given cancer cells but non-essential in other cells, and also those non-essential in cancer genes and essential in other cells. To test GIC for such studies, we randomly selected 10 genes, which include 5 GIC-predicted essential (among the top 25\% GIC scores) but CRISPR/Cas9 non-essential genes in the KBM7 cell line (among the bottom 25\% CRISPR/Cas9 scores) (RIMKLA, ATF7IP, PFN3, HISTIH3J and DDIT4, these genes were named as $\mathrm{GIC}_{\text {essential-Cas }} 9_{\text {nonessential }}$ genes here) and 5 GIC-predicted non-essential (among the bottom 25\% GIC scores) but CRISPR/Cas9 essential genes (among the top 25\% CRISPR/Cas9 scores) (CBWD3, SLC35B1, CCDC33, DPH6 and ZMAT2, these genes were named as GIC $_{\text {nonessential-Cas }} 9_{\text {essential }}$ genes here). Because blood vessel is a basic tissue for cancer cell survival, we evaluate the effects of the ten genes on VSMCs, which one class of key cells for blood vessel. The efficacy of siRNAs treatment on target mRNA levels were first determined by real time PCR assays, revealing that siRNAs treatment significantly reduced the target mRNA levels by about $60-90 \%$ (Figure 4a). For evaluating the essentiality of the 10 target mRNAs on cell survival, they were randomly divided into two groups for siRNAs transfection to reduce experimental errors. It is also noteworthy that the person who performed the experimental validation was blind to the essentiality of these genes during the 
experiments. MTT assays indicated silencing $\mathrm{GIC}_{\mathrm{essential}}-\mathrm{Cas} 9_{\text {nonessential }}$ genes RIMKLA, ATF7IP, PFN3, HIST1H3J and DDIT4 significantly reduced cell viability by $30-50 \%$ (Figure 4b-c). In contrast, among the $\mathrm{GIC}_{\text {nonessential-Cas }} 9_{\text {essential }}$ genes, silencing of DPH6 reduced cell viability by only about $10 \%$, whereas silencing of $C B W D 3, S L C 35 B 1, C C D C 33$ and ZMAT2 had no significant effect on cell viability as evaluated by MTT assays (Figure 4b-c). siRNAs against HIST1H3J and SLC35B1 mRNAs exhibited the highest repression efficacy among all siRNAs. Silencing GIC $_{\text {essential-Cas }}$ nonessential $_{\text {gene } H I S T 1 H 3 J}$ significantly reduced cell viability, whereas silencing $\mathrm{GIC}_{\text {nonessential-Cas9 }}$ essential $_{\text {gene }} \mathrm{SLC} 353 H$ had no significant effect on cell viability of human T/G HA VSMCs. siRNAs against other eight target mRNAs exhibited comparable silencing efficacy (Figure 4a). Overall, these results strongly indicated that the significant reduction in cell viability after silencing $\mathrm{GIC}_{\text {essential-Cas }} 9_{\text {nonessential }}$ genes is not due to repression efficacy of siRNAs (Figure 4a-c).

To further validate the essentiality of $\mathrm{GIC}_{\mathrm{essential}}-\mathrm{Cas} 9_{\text {nonessential }}$ and $\mathrm{GIC}_{\text {nonessential }}-\mathrm{Cas} 9_{\text {essential }}$ genes on cell survival, flow cytometry was performed to analyze the impacts of silencing of two $\mathrm{GIC}_{\text {essential }}-\mathrm{Cas} 9_{\text {nonessential }}$ genes, RIMKLA and $A T F 7 I P$, and two $\mathrm{GICn}_{\text {onessential }}$-Cas9 $9_{\text {essential }}$ genes, $C B W D 3$ and $C C D C 33$, on the apoptosis of human T/G HA VSMCs. Silencing RIMKLA and ATF7IP markedly increased the proportions of apoptotic cells $(51.48 \pm 9.38 \%$ versus $8.08 \pm 1.23 \%$ (Control) for $R I M K L A$, and $40.72 \pm 7.37 \%$ versus $8.08 \pm 1.23 \%$ (Control) for $A T F 7 I P$, respectively, $\mathrm{P}<0.01$ ) (Figure 5a-b). In contrast, silencing $C B W D 3$ slightly increased apoptotic cells $(14.67 \pm 1.02 \%$ versus $8.08 \pm 1.23 \%, \mathrm{P}<0.05)$, and silencing $C C D C 33$ had no statistically significant effect on cell apoptosis when compared with control cells $(13.43 \pm 3.12 \%$ versus $8.08 \pm 1.23 \%, \quad \mathrm{P}>0.05)$ (Figure 5a-b). Importantly, there is also 
significant difference between $\mathrm{GIC}_{\text {essential}}-\mathrm{Cas} 9_{\text {nonessential }}$ and $\mathrm{GIC}_{\text {nonessential }}$-Cas9 $9_{\text {essential }}$ genes

(Figure 5b), confirming the results of the above cell viability assays.

As a comparison, Pheg predicted one of the $5 \mathrm{GIC}_{\text {essential }}-\mathrm{Cas} 9_{\text {nonessential }}$ genes as essential genes and three of the $\mathrm{GIC}_{\text {nonessential-Cas }} 9_{\text {essential }}$ genes as non-essential genes (Supplementary Table S4).

\section{Discussion}

Predicting gene essentiality is an important issue in bioinformatics but computational methods for predicting the essentiality of lncRNAs are still not available. For doing so, here we defined GIC (Gene Importance Calculator) score on the basis of sequence information. Overall, our data validated the high accuracy of GIC score for predicting essential and non-essential genes and lncRNAs. Given that the functions of many human protein-coding genes and lncRNAs are still awaiting exploration, our new method provides an effective strategy for identifying and characterizing new genes and lncRNAs with important functions, which definitely will shed light on the pathogenesis, diagnosis, and therapy of human diseases.

microRNAs (miRNAs) are one class of important small noncoding RNAs. We also tried to test GIC on miRNAs. When evaluating GIC score on miRNAs base on two types of previously presented scores, miRNA conservation score (Wang, et al., 2010) and miRNA disease spectrum width (DSW) score(Qiu, et al., 2012), we found that GIC did not work any more, suggesting the GIC method is not feasible for miRNAs. The reason could be miRNA sequences are very small, so the nucleotide features derived from protein-coding genes are 
not feasible for miRNAs any more. Finally, because miRNAs also have many species (especially human) specific miRNAs and the number of miRNAs with DSW score is still small, the above two scores for miRNAs are not feasible for all miRNAs. So, it is also important to develop new computational methods to predict essential miRNAs.

\section{ACKNOWLEDGEMENTS}

We thank members of Prof. Cui's lab and Prof. Yang's lab for helpful discussions.

\section{FUNDING}

National High Technology Research and Development Program of China [2014AA021102];

National Natural Science Foundation of China [91339106;81422006;81670462]. Funding for open access charge: National High Technology Research and Development Program of China [2014AA021102].

Conlict of interest statement. None declared.

\section{REFERENCES}

Bello, S.M., Smith, C.L. and Eppig, J.T. (2015) Allele, phenotype and disease data at Mouse Genome Informatics: improving access and analysis, Mammalian genome : official journal of the International Mammalian Genome Society, 26, 285-294.

Blomen, V.A., et al. (2015) Gene essentiality and synthetic lethality in haploid human cells, Science, 350, 1092-1096.

Bult, C.J., et al. (2016) Mouse genome database 2016, Nucleic Acids Res, 44, D840-847.

Cheng, J., et al. (2013) A new computational strategy for predicting essential genes, BMC Genomics, 14, 910.

del Rio, G., Koschutzki, D. and Coello, G. (2009) How to identify essential genes from molecular networks?, BMC systems biology, 3, 102.

Deng, J., et al. (2011) Investigating the predictability of essential genes across distantly related organisms using an integrative approach, Nucleic Acids Res, 39, 795-807.

Durinck, S., et al. (2009) Mapping identifiers for the integration of genomic datasets with the R/Bioconductor package biomaRt, Nature protocols, 4, 1184-1191.

Evers, B., et al. (2016) CRISPR knockout screening outperforms shRNA and CRISPRi in identifying essential genes, Nature biotechnology, 34, 631-633.

Gatto, F., et al. (2015) Flux balance analysis predicts essential genes in clear cell renal cell carcinoma 
metabolism, Sci Rep, 5, 10738.

Guo, F.B., et al. (2017) Accurate prediction of human essential genes using only nucleotide composition and association information, Bioinformatics.

Hofacker, I.L., et al. (1994) Fast folding and comparison of RNA secondary structures, Monatsh Chem, 125, 167-188.

Iyer, M.K., et al. (2015) The landscape of long noncoding RNAs in the human transcriptome, Nature genetics, 47, 199-208.

Karolchik, D., et al. (2004) The UCSC Table Browser data retrieval tool, Nucleic Acids Res, 32, D493-D496.

Korona, R. (2011) Gene dispensability, Current opinion in biotechnology, 22, 547-551.

Li, G., et al. (2016) Predicting essential proteins based on subcellular localization, orthology and PPI networks, BMC bioinformatics, 17 Suppl 8, 279.

Li, M., et al. (2015) A Topology Potential-Based Method for Identifying Essential Proteins from PPI Networks, IEEE/ACM transactions on computational biology and bioinformatics, 12, 372-383.

Liang, H. and Li, W.H. (2007) Gene essentiality, gene duplicability and protein connectivity in human and mouse, Trends in genetics: TIG, 23, 375-378.

Lipman, D.J., et al. (2002) The relationship of protein conservation and sequence length, $B M C$ evolutionary biology, 2, 20.

Liu, G., et al. (2015) Gene Essentiality Is a Quantitative Property Linked to Cellular Evolvability, Cell, 163, 1388-1399.

Luo, H., et al. (2014) DEG 10, an update of the database of essential genes that includes both protein-coding genes and noncoding genomic elements, Nucleic Acids Res, 42, D574-D580.

Morgens, D.W., et al. (2016) Systematic comparison of CRISPR/Cas9 and RNAi screens for essential genes, Nature biotechnology, 34, 634-636.

NCBI Resource Coordinators. (2016) Database resources of the National Center for Biotechnology Information, Nucleic Acids Res, 44, D7-D19.

Peng, W., et al. (2015) UDoNC: An Algorithm for Identifying Essential Proteins Based on Protein Domains and Protein-Protein Interaction Networks, IEEE/ACM transactions on computational biology and bioinformatics, 12, 276-288.

Peters, J.M., et al. (2016) A Comprehensive, CRISPR-based Functional Analysis of Essential Genes in Bacteria, Cell, 165, 1493-1506.

Qiu, C., Chen, G. and Cui, Q. (2012) Towards the understanding of microRNA and environmental factor interactions and their relationships to human diseases, Sci Rep, 2, 318.

Robin, X., et al. (2011) pROC: an open-source package for R and S+ to analyze and compare ROC curves, BMC bioinformatics, 12, 77.

Sauvageau, M., et al. (2013) Multiple knockout mouse models reveal lincRNAs are required for life and brain development, eLife, 2, e01749.

Seringhaus, M., et al. (2006) Predicting essential genes in fungal genomes, Genome research, 16, 1126-1135.

Stark, C., et al. (2006) BioGRID: a general repository for interaction datasets, Nucleic Acids Res, 34, D535-D539.

Tzelepis, K., et al. (2016) A CRISPR Dropout Screen Identifies Genetic Vulnerabilities and Therapeutic Targets in Acute Myeloid Leukemia, Cell reports, 17, 1193-1205.

Wan, Y., et al. (2014) Landscape and variation of RNA secondary structure across the human 
transcriptome, Nature, 505, 706-709.

Wang, D., et al. (2010) Human microRNA oncogenes and tumor suppressors show significantly different biological patterns: from functions to targets, PLoS One, 5.

Wang, T., et al. (2015) Identification and characterization of essential genes in the human genome, Science, 350, 1096-1101.

Wang, T., et al. (2015) Identification and characterization of essential genes in the human genome, Science, 350, 1096-1101.

Wang, T., et al. (2014) Genetic screens in human cells using the CRISPR-Cas9 system, Science, 343, 80-84.

Yates, A., et al. (2016) Ensembl 2016, Nucleic Acids Res, 44, D710-D716.

Zhao, Y., et al. (2016) NONCODE 2016: an informative and valuable data source of long non-coding RNAs, Nucleic Acids Res, 44, D203-D208.

Zhao, Y., et al. (2016) NONCODE 2016: an informative and valuable data source of long non-coding RNAs, Nucleic Acids Res, 44, D203-208.

Zhou, Y., et al. (2014) High-throughput screening of a CRISPR/Cas9 library for functional genomics in human cells, Nature, 509, 487-491.

Zhu, S., et al. (2016) Genome-scale deletion screening of human long non-coding RNAs using a paired-guide RNA CRISPR-Cas9 library, Nature biotechnology. 


\section{Figure Legends}
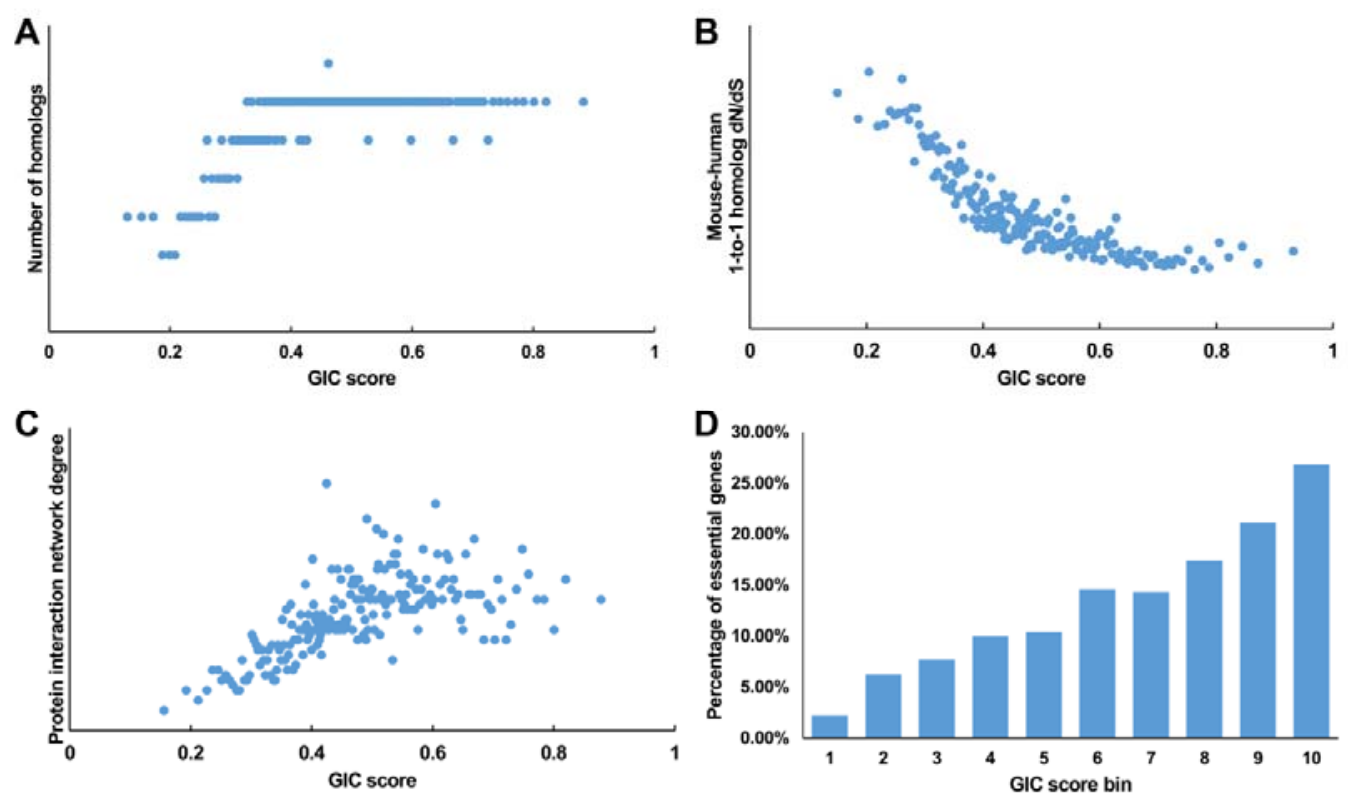

Figure 1. Correlation of GIC score with known measurements of essentiality in human genes.

(a) Genes with higher GIC scores tend to have more homologs across species. (b) Genes with higher GIC scores tend to have slower evolutionary rate as measured by $\mathrm{dN} / \mathrm{dS}$ ratio. (c) Proteins encoded by genes with higher GIC scores tend to have higher degrees in protein interaction network. (d) The percentage of human essential genes increases with GIC score. 

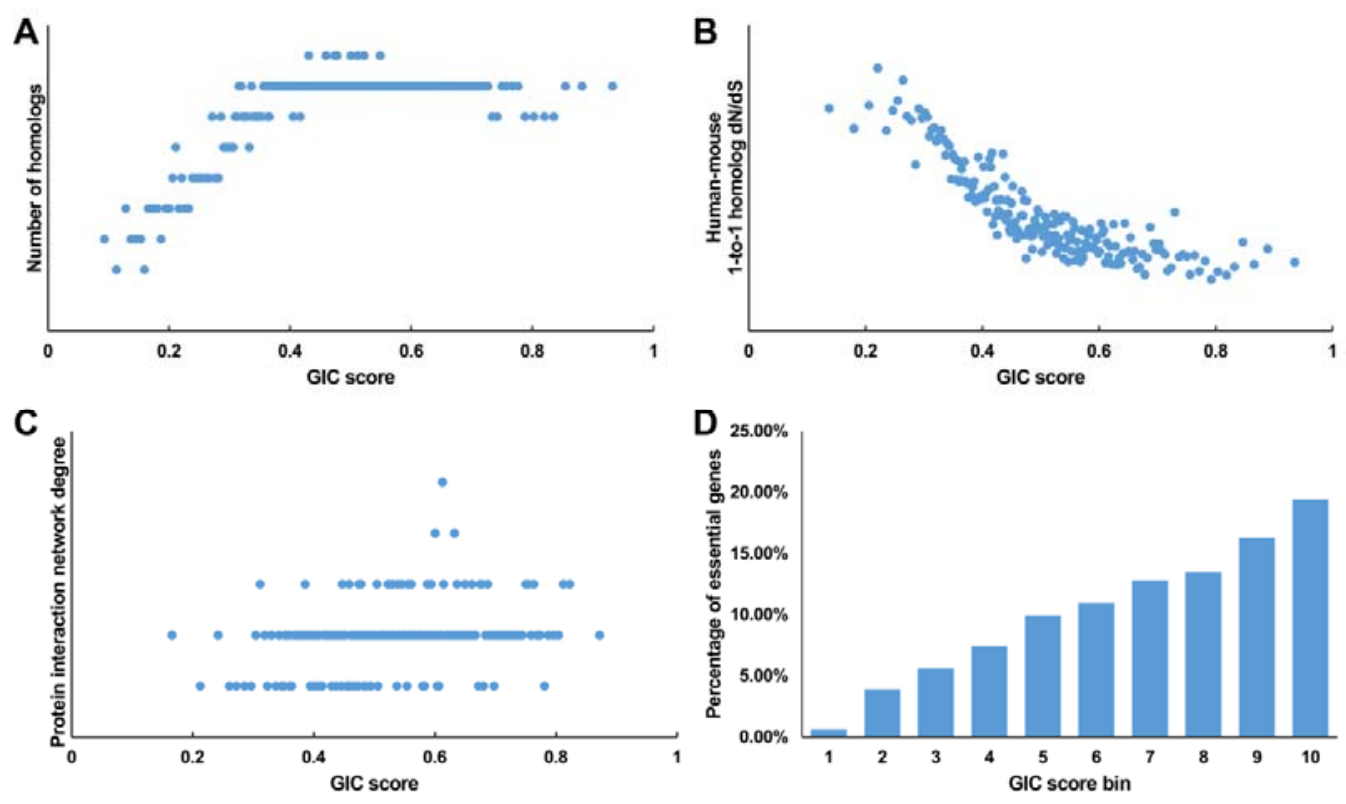

Figure 2. Correlation of GIC score with known measurements of essentiality in mouse genes.

(a) Genes with higher GIC scores tend to have more homologs across species. (b) Genes with higher GIC scores tend to have slower evolutionary rate as measured by $\mathrm{dN} / \mathrm{dS}$ ratio. (c) Proteins encoded by genes with higher GIC scores tend to have higher degrees in protein interaction network. (d) The percentage of human essential genes increases with GIC score. 

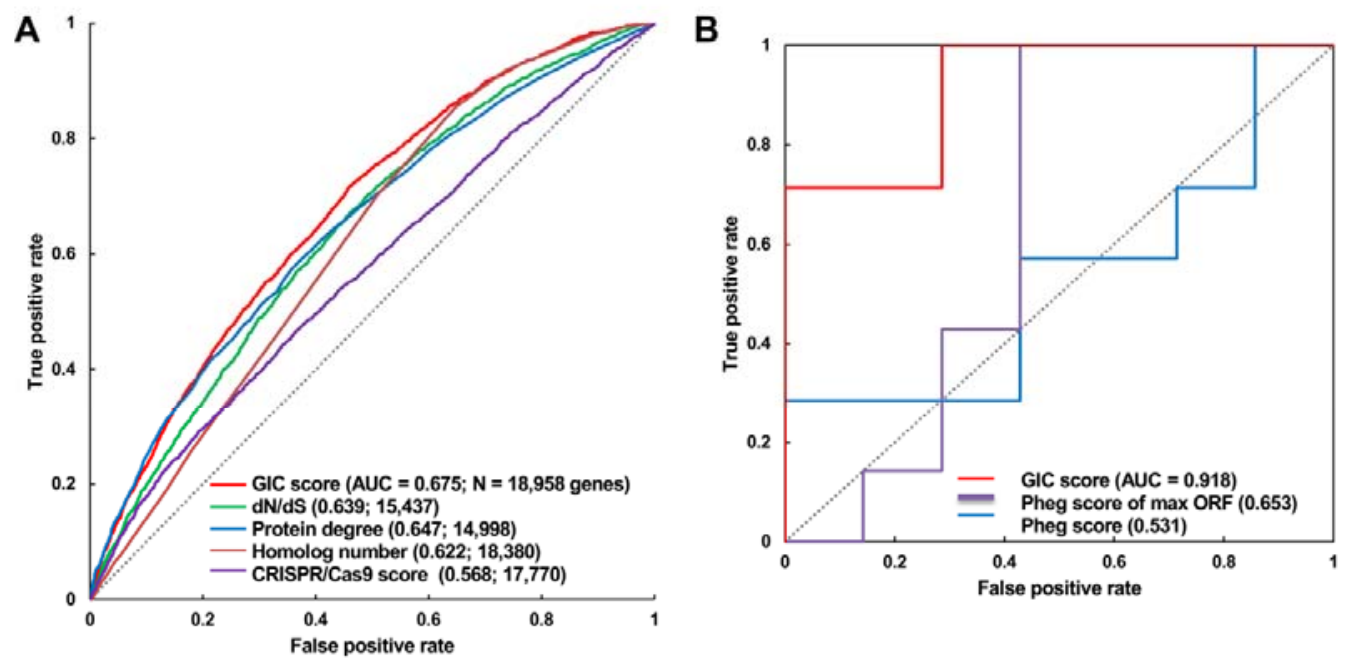

Figure 3. Validation of GIC score. (a) ROC curves illustrating the results from human essential gene prediction analysis. (b) ROC curves illustrating the results of essentiality prediction in an independent mouse lncRNA dataset. 
A

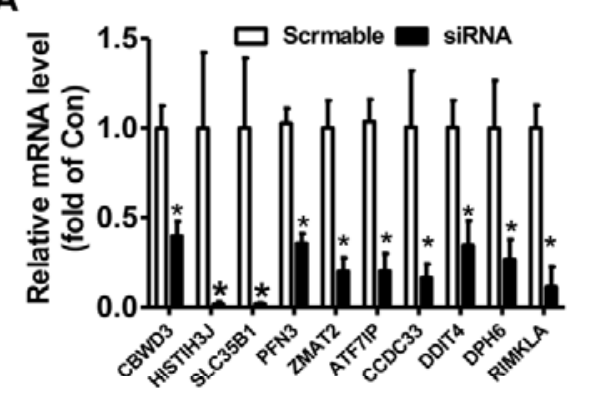

B

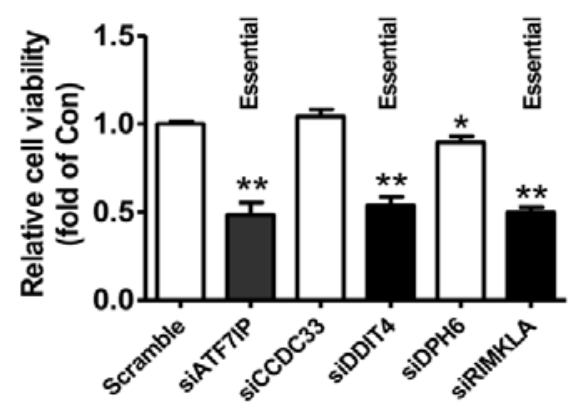

C

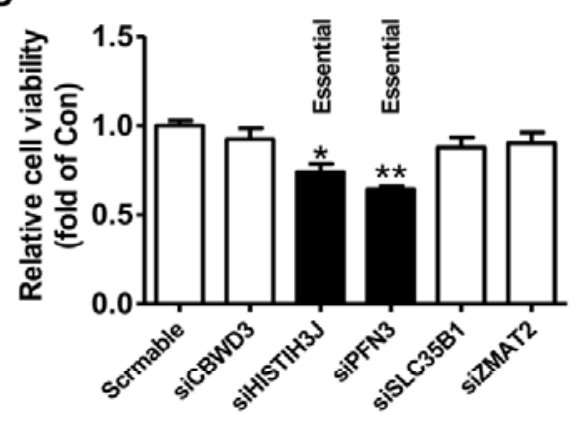

Figure 4. siRNA-mediated silencing of target mRNAs on the cell viability of human T/G

HA VSMCs. (a) The efficacy of siRNA treatment on the repression of target mRNA levels.

The target mRNA levels were analyzed by real time PCR assays at 48 hours post siRNA transfection. $\mathrm{N}=6, * \mathrm{P}<0.05$ versus control cells transfected with scrambled siRNAs. (b-c) Silencing of target mRNAs on the cell viability. At 48 hours post siRNA transfection, cell viability was determined using MTT assay as described in experimental procedure. In every experiment, 3-4 parallel observations were set for each siRNA mixture. In panels b and c, GIC-predicted essential genes but CRISPR/Cas9 non-essential genes were presented as fill bars, whereas GIC-predicted non-essential genes but CRISPR/Cas9 essential genes were presented as blank bars. $\mathrm{N}=4, * \mathrm{P}<0.05, * * \mathrm{P}<0.01$ versus control cells transfected with scrambled siRNAs. 

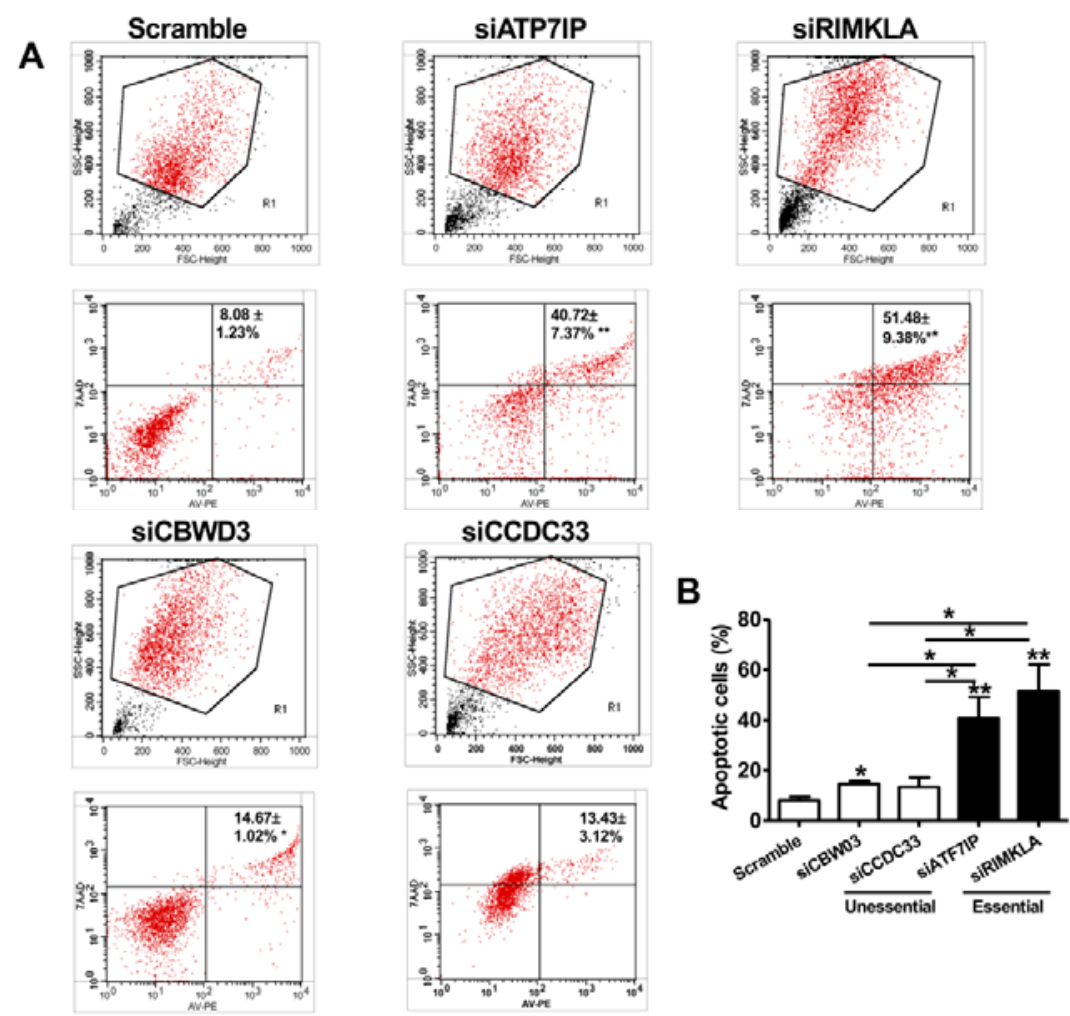

Figure 5. Flow cytometry analysis of apoptosis of human T/G HA VSMCs. The essentiality of two GIC-predicted essential genes but CRISPR/Cas9 non-essential genes and two GIC-predicted non-essential genes but CRISPR/Cas9 essential genes in cell survival were selected for further validation, respectively. The cells were transfected with siRNAs against target mRNAs or scrambled siRNA, and the apoptosis was determined by flow cytometry analysis at 48 hours later. (a) Representative images of flow cytometry analysis. (b) Quantitative data of apoptosis determined by flow cytometry. $\mathrm{N}=4, * \mathrm{P}<0.05, * * \mathrm{P}<0.01$ versus control cells treated with scrambled siRNAs or between two indicated groups. 

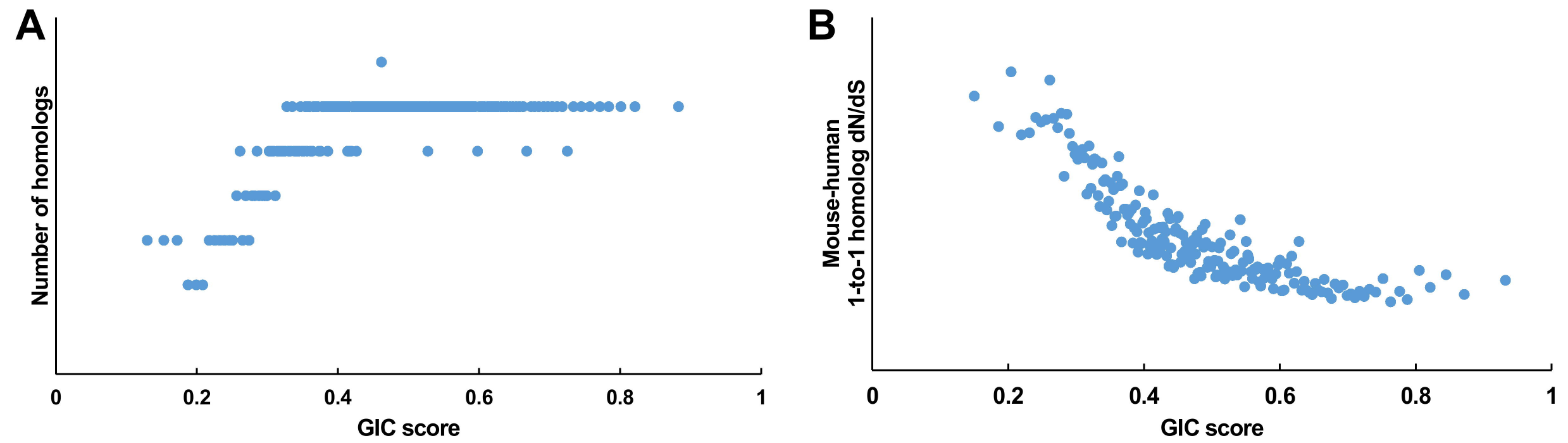

C
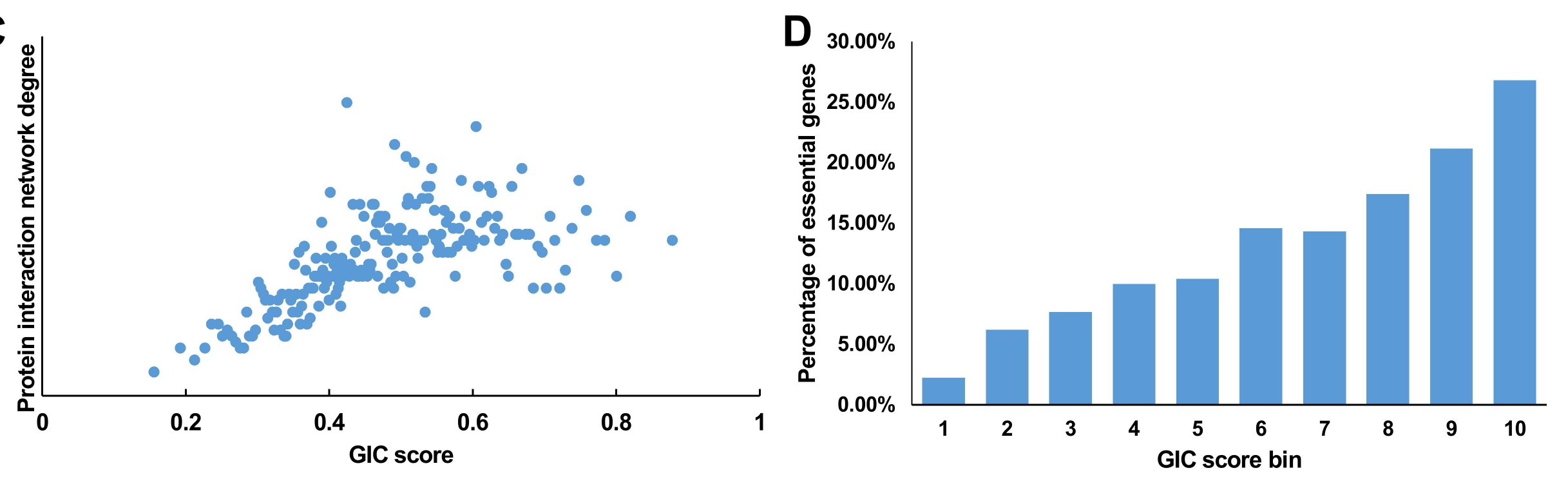

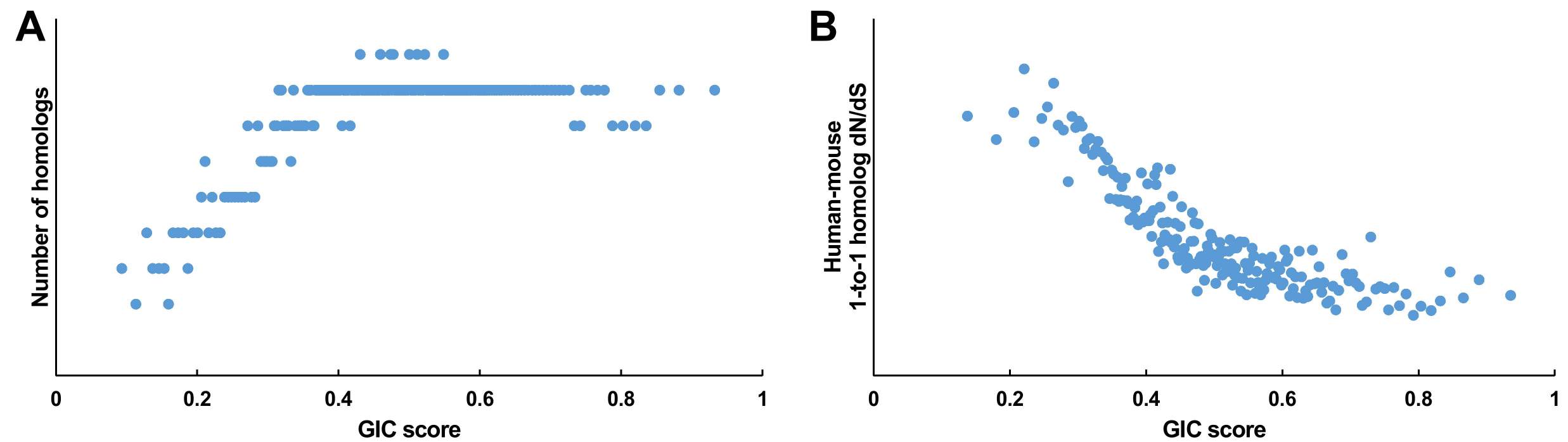

C

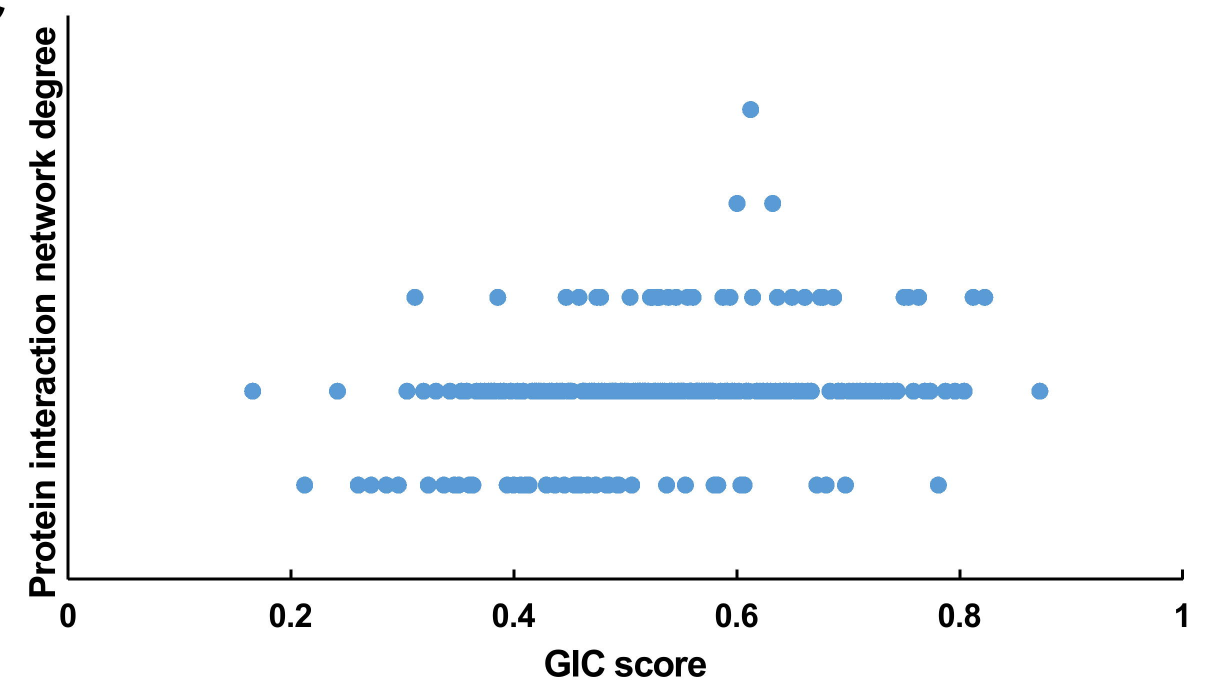

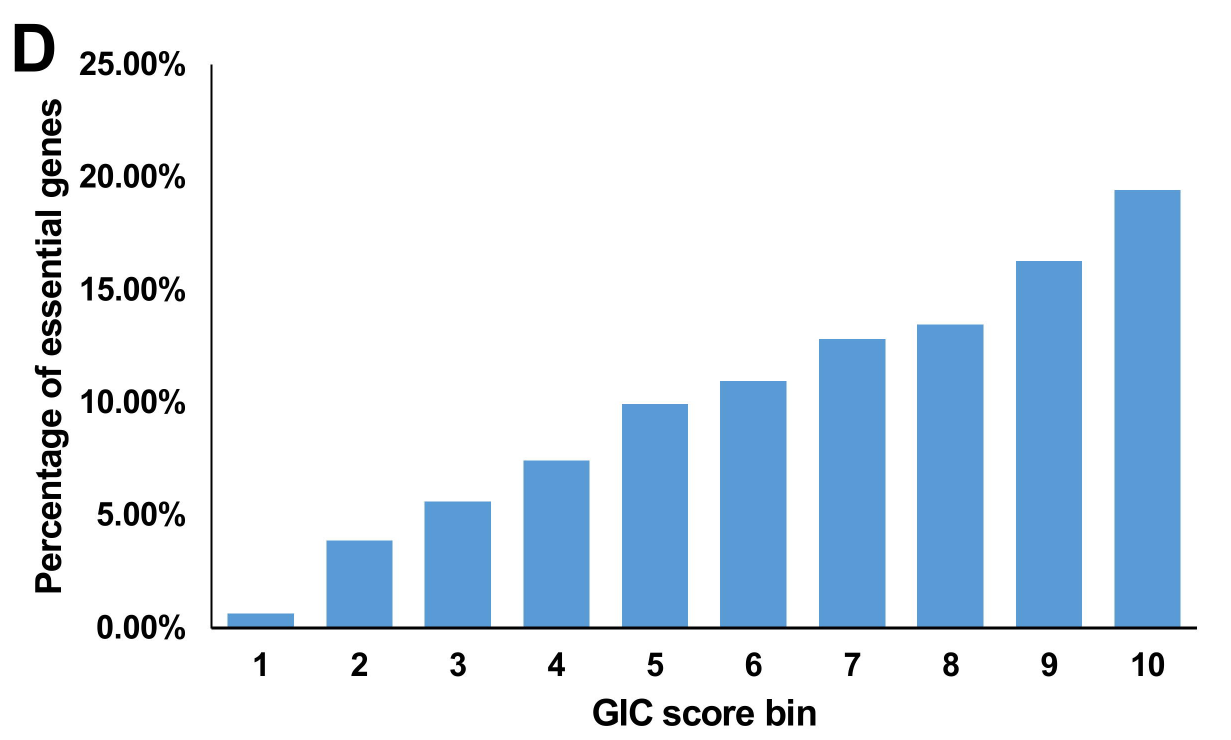



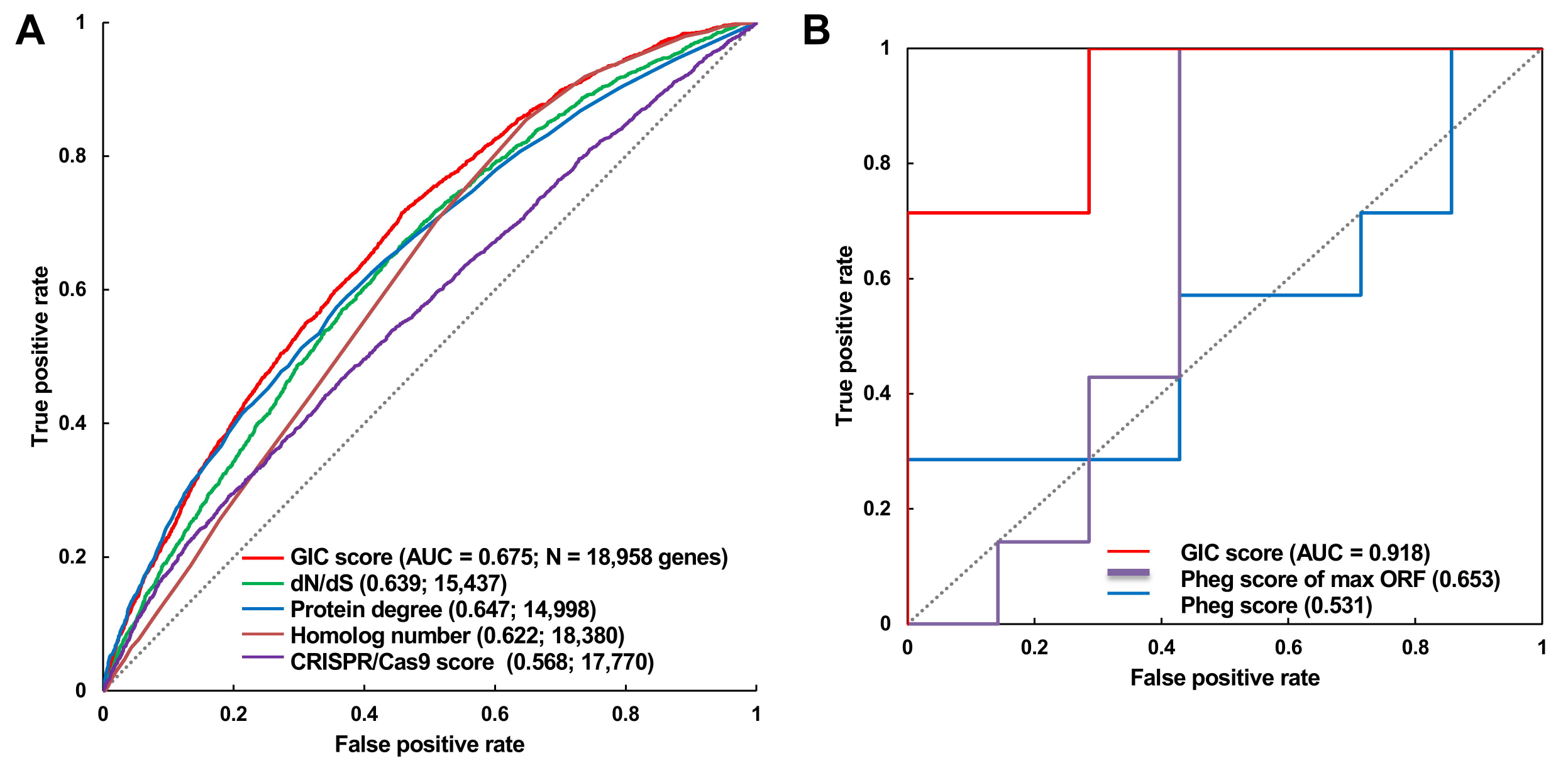
A

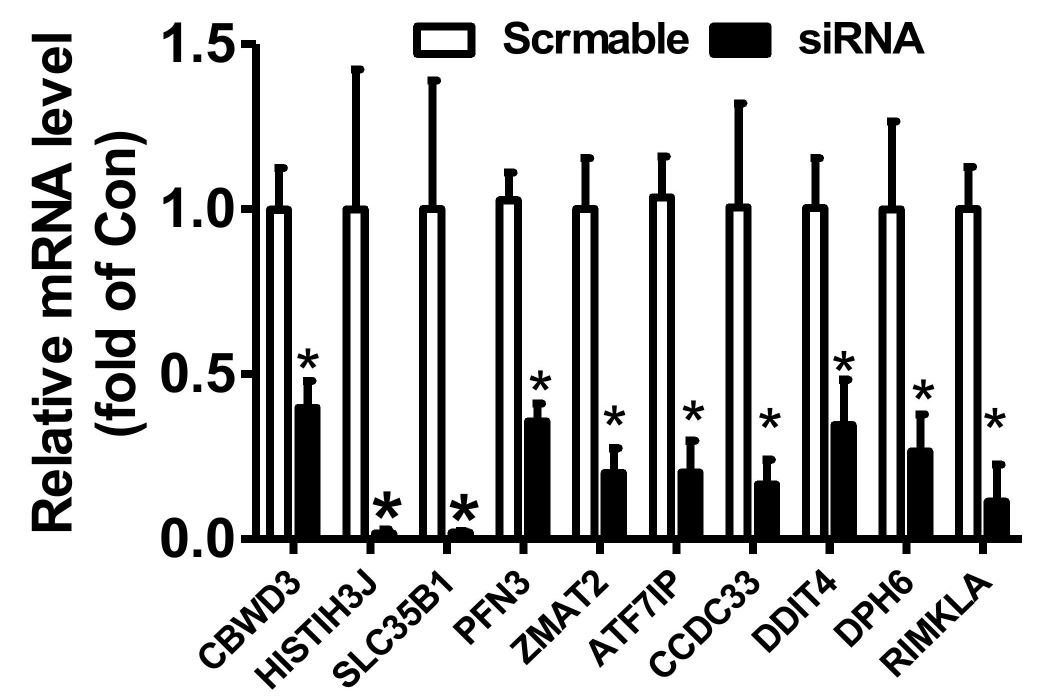

B

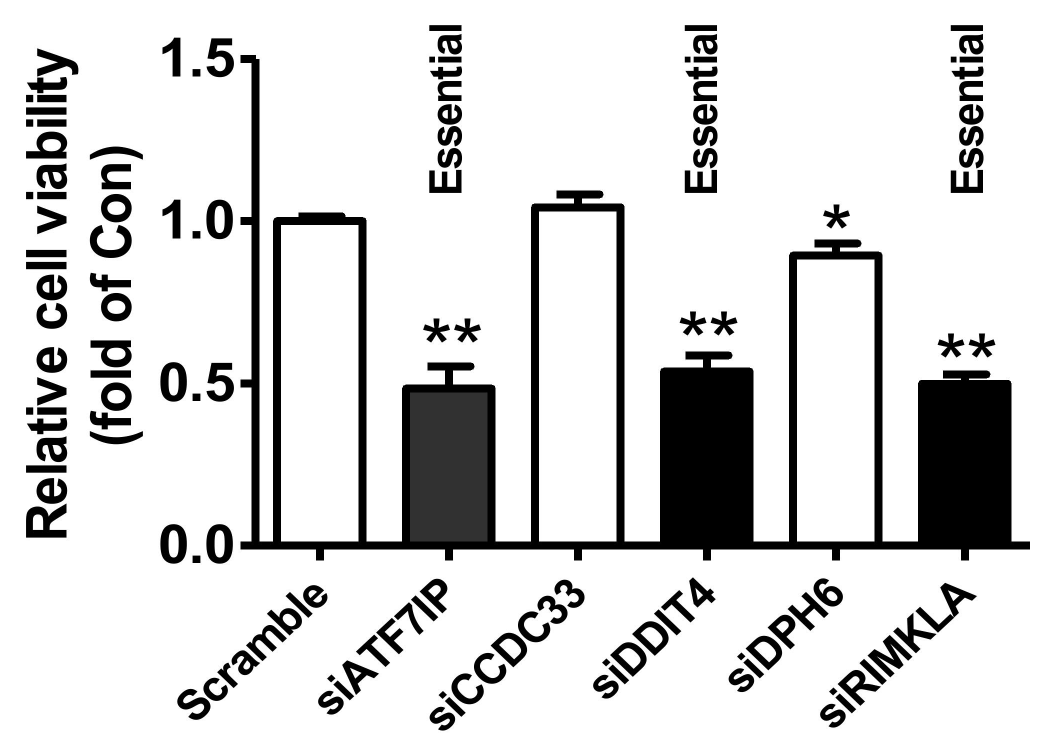

C

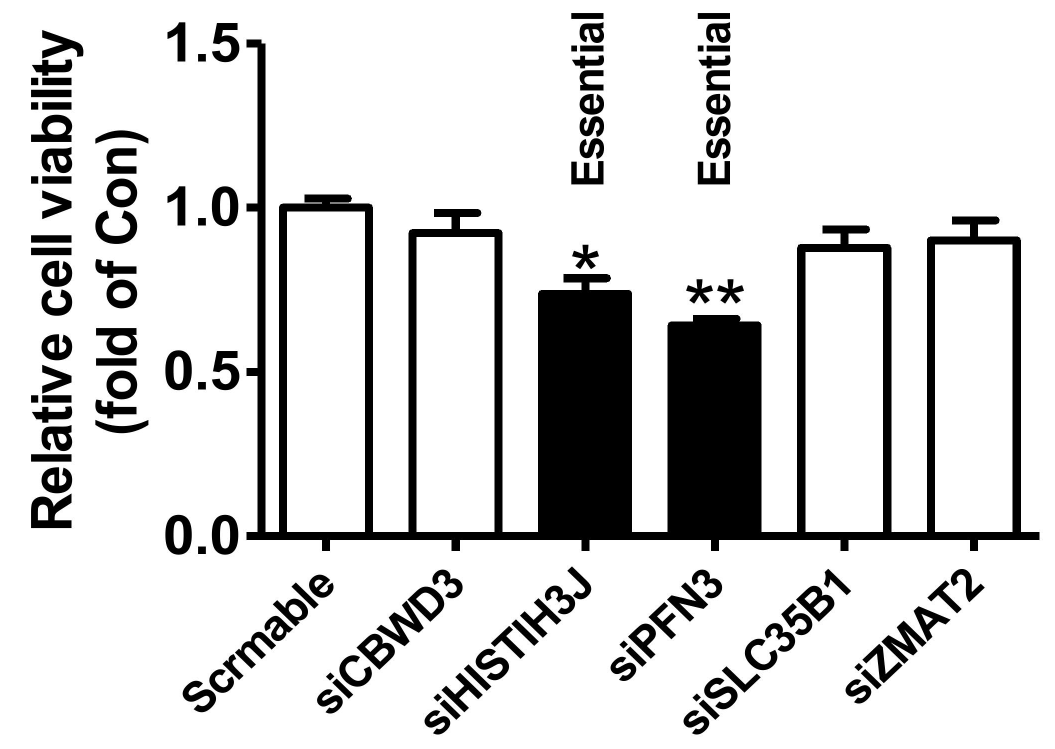



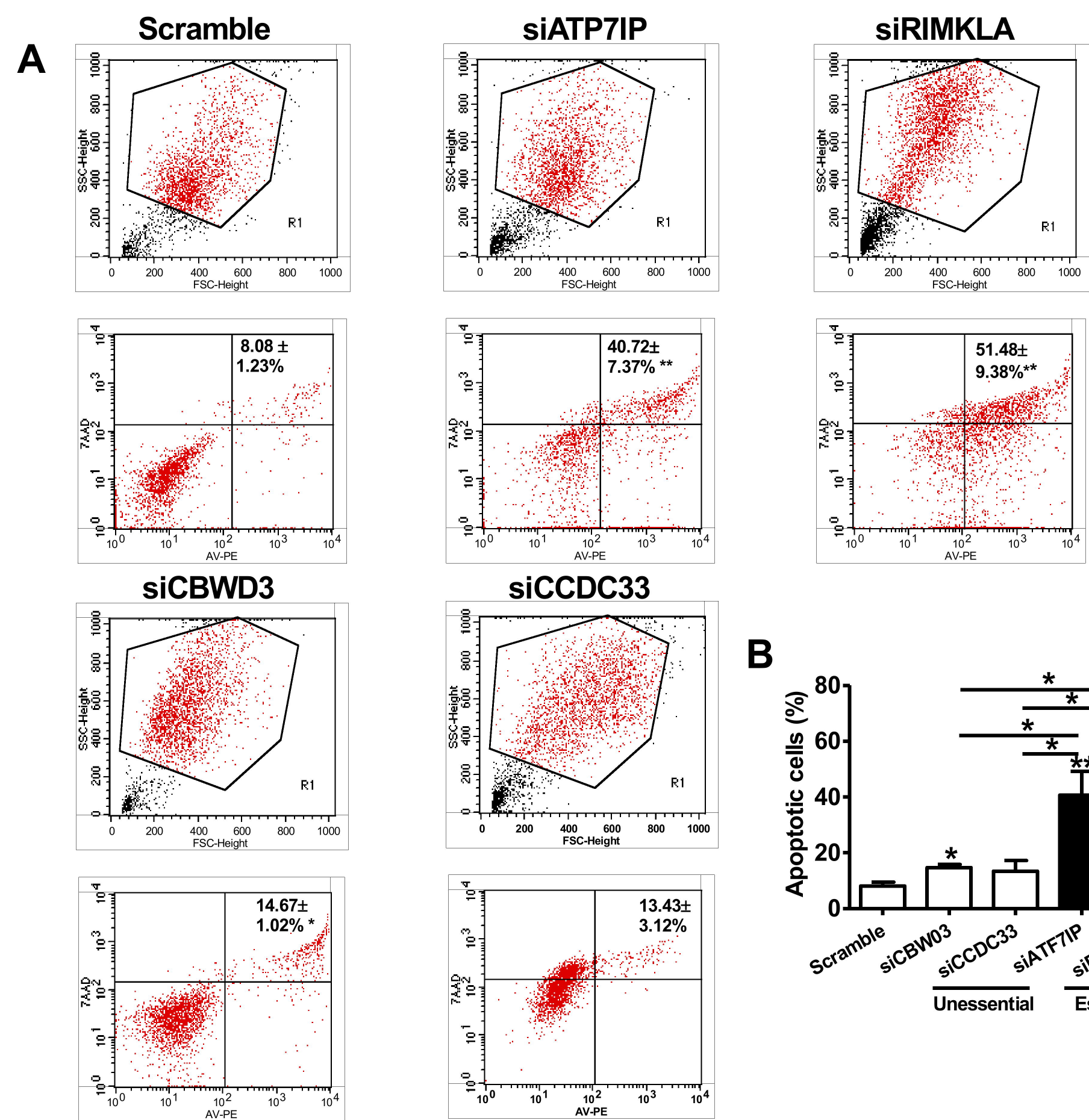

B

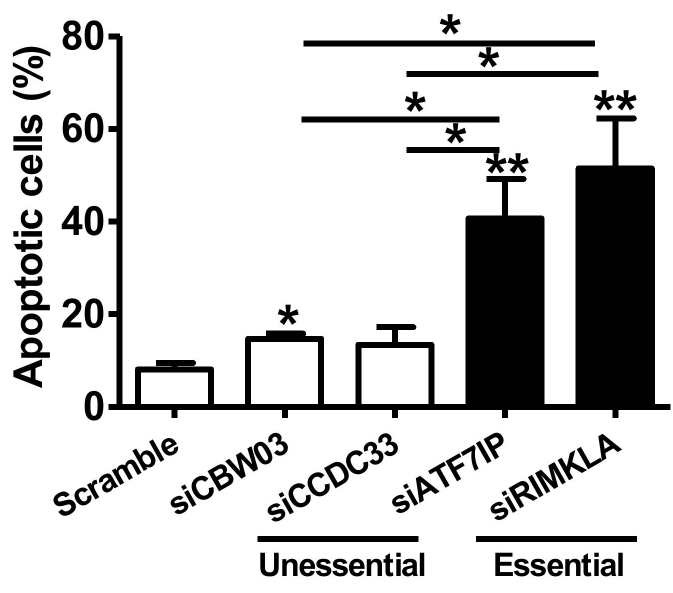

